\title{
AOP2 (Antioxidant Protein 2): Structure and Function of a Unique Thiol-Specific Antioxidant
}

Shelley A. Phelan

Fairfield University, sphelan@fairfield.edu

Follow this and additional works at: https://digitalcommons.fairfield.edu/biology-facultypubs

This is a copy of an article published in the "Antioxidants \& Redox Signaling" (C) WINTER 1999

[copyright Mary Ann Liebert, Inc.]; "Antioxidants \& Redox Signaling" is available online at: http://www.liebertonline.com.

\section{Peer Reviewed}

\section{Repository Citation}

Phelan, Shelley A., "AOP2 (Antioxidant Protein 2): Structure and Function of a Unique Thiol-Specific Antioxidant" (1999). Biology Faculty Publications. 2.

https://digitalcommons.fairfield.edu/biology-facultypubs/2

\section{Published Citation}

Shelley A. Phelan. Antioxidants \& Redox Signaling. Winter 1999, 1(4): 571-584.

This item has been accepted for inclusion in DigitalCommons@Fairfield by an authorized administrator of DigitalCommons@Fairfield. It is brought to you by DigitalCommons@Fairfield with permission from the rightsholder(s) and is protected by copyright and/or related rights. You are free to use this item in any way that is permitted by the copyright and related rights legislation that applies to your use. For other uses, you need to obtain permission from the rights-holder(s) directly, unless additional rights are indicated by a Creative Commons license in the record and/or on the work itself. For more information, please contact digitalcommons@fairfield.edu. 


\title{
Commentary
}

\section{AOP2 (Antioxidant Protein 2): Structure and Function of a Unique Thiol-Specific Antioxidant}

\author{
SHELLEY A. PHELAN
}

\begin{abstract}
The accumulation of reactive oxygen species (ROS) in response to extracellular signals or intracellular biochemical processes can be regulated by the coordinate action of many antioxidant proteins. Because moderate levels of ROS can act as intracellular messengers in many of these processes, this modulation is critical for the transduction of specific signals. The thiol-specific antioxidant (TSA) family is a highly conserved group of enzymes that can reduce hydroperoxides in the presence of a thiol-containing electron donor. AOP2 (antioxidant protein 2) is a newly described member that shows significant evolutionary conservation between many different organisms. The protein contains three motifs that are highly conserved within the TSA family, including a cysteine residue that is the active site of oxidation for this class of proteins. Although AOP2 possesses TSA activity, it has several unique characteristics, including the absence of a second cysteine residue that is conserved in all other TSA proteins, the presence of a unique carboxy-terminal domain, and a demonstrated phospholipase activity. Furthermore, AOP2 shows conservation of several amino acids important in dimer formation and active site configuration that are not found in the other family members. Together, these data strongly suggest that AOP2 is a novel thiol-dependent antioxidant that functions to scavenge particular hydroperoxides in the cell and mediate specific signals. There is also evidence supporting a role for AOP2 in certain disease processes including atherosclerosis. Further evaluation of this protein and its substrate specificity will likely shed light on its precise role in cellular oxidant defense, signal transduction and pathogenesis. Antiox. Redox Signal. 1, 571-584.
\end{abstract}

\section{INTRODUCTION}

$\mathbf{O}$ VER THE PAST SEVERAL YEARS, many investigations have focused on understanding how cells respond to their environment and cope with a wide variety of environmental insults. These stress-induced responses are quite diverse in nature, including the typical inflammatory response following infection and wound repair after cellular injury. Although these events are involved in different physiological processes, they share many of the same biochemical mechanisms. One common occur- rence is the generation of reactive oxygen species (ROS), including superoxide anion $\left(\mathrm{O}_{2}{ }^{--}\right)$, hydroxyl radical (HO), nitric oxide (NO*), and hydrogen peroxide $\left(\mathrm{H}_{2} \mathrm{O}_{2}\right)$. These are highly reactive molecules that can directly damage membranes, proteins, and DNA in the cell and, at certain concentrations, can regulate specific signal transduction pathways by acting as intracellular messengers. In addition to their known role in immunological host defense, ROS are generated as a by-product during normal metabolic activity, such as cellular respiration, and are also thought to play an integral

Department of Biology, Fairfield University, Fairfield, CT 06430. 
role in many pathophysiological processes including aging, heart disease, and cancer. In an effort to combat the accumulation of ROS, particular defense mechanisms have evolved to aid in the cell's response to oxidative stress. These include a diverse group of antioxidant molecules, each of which is thought to have a specific function in the stress response. The continual balance between oxidants and antioxidants in the cell is believed to be a critical factor in the regulation of many biological processes.

Introduction to the thiol-specific antioxidant family

Several antioxidant proteins have been identified that play essential roles in "detoxifying" free radicals. The most well-characterized groups include catalases, glutathione peroxidases, and superoxide dismutases. However, other antioxidant proteins have recently emerged, although their specific functions in the cell remain undetermined. One newly described family is the thiol-specific antioxidant (TSA) family, which shows no significant homology with previously identified antioxidant proteins. These proteins, also named rehydrins, thioredoxin-dependent peroxide reductases (TPx), and recently renamed peroxiredoxins (PRx) (Chae et al., 1994b; Rhee and Chae, 1994), comprise a family of enzymes that reduce hydroperoxides using thiol-containing electron donors. The family was first described in yeast with the isolation and characterization of yeast TSA (TPX), a protein capable of protecting cells from oxidative damage induced by metal catalyzed oxidative systems in the presence of diothiothreitol (DTT), but not in the presence of ascorbate (Kim et al., 1988). This finding suggested a thiol-specific function for this protein. Since then, many homologous proteins have been identified that are highly conserved in prokaryotes and eukaryotes, and possess TSA activity (Chae et al., 1994b). Thus, this group of proteins likely represents a new family of important antioxidant molecules.

Despite apparent sequence similarities, current literature on this family is confusing due to nonconsensus of protein function, inconsistencies in nomenclature, and ambiguities in subfamily classification. Assortment of the mammalian TSA family members by nucleotide and protein sequence comparison was performed, and is shown in Table 1 along with

Table 1. Organization of the Mammalian Thiol-Specific Antionidant Family

\begin{tabular}{|c|c|c|c|c|c|}
\hline & Group I & Group II & Group III & Group IV & Group V \\
\hline Human & $\begin{array}{l}\text { PAG, TDX2 } \\
\text { (Prosperi et al., 1993) } \\
\text { NKEFA } \\
\text { (Shau et al., 1994) }\end{array}$ & $\begin{array}{l}\text { TSA, TDX1 } \\
\text { (Lim et al., 1994) } \\
\text { NKEFB } \\
\text { (Shau et al., 1994) } \\
\text { TPXII } \\
\text { (Zhang et al., 1997) }\end{array}$ & $\begin{array}{l}\text { MER5 } \\
\text { (Tsuji et al., 1995) }\end{array}$ & $\begin{array}{l}\text { AOE372, TDXN } \\
\text { (Jin et al., 1997) }\end{array}$ & $\begin{array}{l}\text { KRG-1 } \\
\text { (Frank et al., 1997) } \\
\text { aiPLA } 2 \\
\text { (Kim et al., 1997) } \\
\text { AOP2 } \\
\text { (Iakoubova } \\
\quad \text { et al., 1997) }\end{array}$ \\
\hline \multirow[t]{2}{*}{ Mouse } & $\begin{array}{l}\text { MSP23 } \\
\text { (lshii et al., 1993) }\end{array}$ & $\begin{array}{l}\text { TSA, TDPX1 } \\
\text { (Ichimiya et al., 997) }\end{array}$ & $\begin{array}{l}\text { MER5, TDXM } \\
\text { (Yamamoto } \\
\quad \text { et al., 1999) }\end{array}$ & $\begin{array}{l}\text { AOE372, TDXN } \\
\text { (jin et al., direct } \\
\text { GenBank } \\
\text { submission) }\end{array}$ & $\begin{array}{l}\text { AOP2 } \\
\text { (lakoubova } \\
\quad \text { et al., 1997) }\end{array}$ \\
\hline & $\begin{array}{l}\text { OSF3 } \\
\text { (Kawai et al., 1994) }\end{array}$ & & $\begin{array}{l}\text { AOP1 } \\
\text { (Tsuji et al., 1995) }\end{array}$ & & $\begin{array}{l}\text { GPx } \\
\text { (Munz et al., 1997) }\end{array}$ \\
\hline Rat & $\begin{array}{l}\text { HBP23 } \\
\text { (Iwahara et al., 1995) } \\
\text { PrxI } \\
\text { (Matsumoto } \\
\quad \text { et al., 1999) }\end{array}$ & $\begin{array}{l}\text { TSA, TDX1 } \\
\text { (Chae et al., 1994b) } \\
\text { PrxII } \\
\text { (Matsumoto } \\
\quad \text { et al., 1999) }\end{array}$ & $\begin{array}{l}\text { PRx III } \\
\text { (Matsumoto } \\
\quad \text { et al., 1999) }\end{array}$ & $\begin{array}{l}\text { PRx IV } \\
\text { (Matsumoto } \\
\quad \text { et al., 1999) }\end{array}$ & $\begin{array}{l}\text { aiPLA } \\
\text { (Peshenko } \\
\quad \text { et al., 1996) }\end{array}$ \\
\hline Yeast & $?$ & $\begin{array}{l}\text { TSA1, TPx } \\
\text { (Chae et al., 1993) }\end{array}$ & $?$ & $?$ & $\begin{array}{l}\text { YBG4 } \\
\text { (Scherens } \\
\text { et al., 1993) }\end{array}$ \\
\hline
\end{tabular}

Based on amino acid sequence comparison, TSA proteins from human, mouse, and rat have been assorted into five groups. Corresponding yeast proteins that have been identified are shown. References for reported proteins are also included. 
the corresponding references. The first family member was named $O s f 3$ and $M s p 23$ in mouse, and PAGA and NKEFA in human. It encodes a 199-amino-acid cytoplasmic protein that is induced by stress in macrophages, and is thought to be involved in cell proliferation and differentiation. The second member is named Tsa and Tdpx1 in mouse, and TSA, TDX1, TPxII, and NKEFB in human. It encodes a 198-aminoacid protein localized to the cytosol that is also thought to be associated with cellular stress response. The third family member is Mer5 (renamed Aop1), first cloned from mouse and later from human as TDXM. It encodes a 257-aminoacid protein expressed in mitochondria that may function, along with other mitochondrial antioxidants, to scavenge ROS from the electron transport chain. The newest family member is $A O E 372$, encoding a 271-amino-acid protein that was isolated as a proliferationassociated gene (PAG)-associated protein from human and was recently cloned from mouse and rats. There are conflicting reports on the cellular localization of AOE372, which was first identified as a cytosolic heterodimer (Jin et al., 1997), but was also detected in conditioned medium from Jurkat and HL60 cells (Haridas et al., 1998) and recently shown to be a secreted protein (Matsumoto et al., 1999). Due to the demonstrated ability of these four proteins to reduce $\mathrm{H}_{2} \mathrm{O}_{2}$ using thioredoxin as the electron donor (Jin et al., 1997; Kang et al., 1998b), they have been renamed PRx I-IV, respectively. Finally, the most divergent member of the TSA family is Aop2 (antioxidant protein 2) (lakoubova et al., 1997), also named GPx in mouse (Munz et al., 1997) and aiPLA $A_{2}$ (Kim et al., 1997) and KRG-1 (Frank et al., 1997) in human. This 224 -amino-acid protein shares only $31 \%$ identity and $44 \%$ homolog with its closest family member. Although different in amino acid composition, one unifying feature of the family is the presence of a cysteine residue in the amino terminus that is conserved in all TSA proteins and has been shown to be the active site for enzymatic activity (Chae et al., 1994c). In addition, all members except AOP2 have a second conserved cysteine in their carboxyl terminus that corresponds to Cys170 in yeast TSA, dividing the TSA family into the 2-Cys and 1Cys subfamilies. Consequently, AOP2 has also been named 1-Cys PRx to distinguish it from other peroxiredoxins (Kang et al., 1998a). Although the function of 2-Cys antioxidants has begun to be elucidated, the role of AOP2 is not yet understood and it will be the focus of this commentary.

\section{Aop2: gene and protein}

Murine Aop 2 was first isolated from liver and kidney as a cDNA corresponding to a protein variant between the $\mathrm{C} 57 \mathrm{BL} / 6 \mathrm{~J}$ and $\mathrm{DBA} / 2 \mathrm{~J}$ strains of mice (Iakoubova et al., 1997). Aop 2 has also been identified in many different species, including human, pig, mouse, rat, nematode, barley, rice, moss, arabidopsis, yeast, and bacteria. ${ }^{*}$ Comparison of the encoded protein sequences reveals a high degree of identity, with the human protein sharing $89 \%$ identity and $93 \%$ similarity with murine APO2, and $48 \%$ identity and $63 \%$ similarity with yeast AOP2. We cloned the corresponding gene in mouse and determined it to have five exons and four introns, spanning approximately $11 \mathrm{~kb}$ (Phelan et al., 1998). Analysis of the proximal promoter reveals several putative binding sites for SP1, and consensus sequences for other transcription factors including SREBP, HSF, Pit1, c-Jun, and c-Myc (Phelan et al., 1998; Lee et al., 1999). Lee et al. have shown specific binding of Sp1 and Pit-1a to these elements by gel-shift assays, suggesting a possible role in Aop 2 regulation. The murine Aop2 gene was localized to chromosome 1 at $83.6 \mathrm{cM}$ position on the MGD/ Chromosome Committee maps (Iakoubova et al., 1997), and we have since used a large backcross to fine map the gene to a $0.05 \mathrm{cM}$ interval between D1Mit266 and D1Mit424 (Beier, Phelan, and Paigen, manuscript in prepara-

\footnotetext{
*Accession numbers for the various AOP2 protein sequences used for consensus determination are: Homo sapiens (human) (D14662); Bos taurus (pig) (AAC84043); Mus musculus (mouse) (Y12883); Rattus norvegicus (rat) (AAB66341); Onchocerca volvulus (nematode) (U31052); Hordeum vulgare (barley) (X76605); Oryza sativa (rice) (D3917); Arabidopsis thaliana (thale cress) (O04005); Bromus secalinus (Seed plant) (P52571); Tortula ruralis (moss) (U40818); Saccharomyces cerevisiae (yeast) (P34227); Proteobacterin (AF075709); and Archaebacteria (U36479).
} 
tion). This region corresponds to chromosome 1 in humans, yet neither the human gene nor any other Aop 2 gene has been mapped to date.

The full-length mouse cDNA is approximately $1.45 \mathrm{~kb}$ (Munz et al., 1997), which corresponds to the transcript size observed by our group and others by Northern blot (Munz et al., 1997; Lee et al., 1999). In contrast, the full-length cDNA for rat, bovine, and human is $1.4 \mathrm{~kb}$ (Andreeva et al., 1998), $1.68 \mathrm{~kb}$ (Fisher et al., 1999), and $1.65 \mathrm{~kb}$ (Kim et al., 1997), respectively. The difference between rat/mouse and bovine/human sequences is mostly in the Iength and content of the $3^{\prime}$ untranslated region (UTR). The transcript size detected by Northern blot is 1.7 $\mathrm{kb}$ in rat and $1.9 \mathrm{~kb}$ in human, suggesting either that the published cDNAs are incomplete, or there may be possible alternative splicing or processing modifications of the transcript. Analysis of the EST Database suggests that Aop 2 may be expressed ubiquitously, although its tissue distribution is variable. In mouse and rat, the highest levels are found in lung, moderate levels in heart, liver, and kidney, lower levels in brain, spleen, and stomach, and lowest levels in intestine (Munz et al., 1997; Kim et al., 1998; Lee et al., 1999). The encoded protein is 224 amino acids in length, with a predicted size of $25 \mathrm{kD}$, corresponding to the size observed by two-dimensional gel electrophoresis reported by Iakoubova et al. In vitro transcription/translation of AOP2 produces a protein of approximately the predicted size, as well as a $32-\mathrm{kD}$ band in mouse and rat. The discrepancy is likely due to an in vitro artifact, since immunoblots of endogenous AOP2 in rat tissues reveal only a $26-\mathrm{kD}$ product (Kim et al., 1998). The protein expression pattern has only been reported for the rat thus far, in which AOP2 was detected exclusively in the lung (consistent with its highest mRNA expression) (Kim et al., 1998). However, the lack of detectable AOP2 expression in other tissues is likely due to insensitivity of their assay, since the mRNA tissue distribution indicates widespread expression, and we have detected AOP2 protein in the liver (data not shown). Finally, the cellular localization of the human protein was determined to be cytoplasmic in COS cells transiently transfected with a recombinant influenza virus hemagglutinin (IVH)-tagged construct (Frank et al., 1997), and by subcellular localization of recombinant human AOP2 (Kang et al., 1998a). Together, these data strongly suggest an important cytosolic role for AOP2 in many different tissues.

It is clear that Aop2 belongs to a multigene family, since many studies have reported multiple bands detected by Southern blot (Frank et al., 1997; Munz et al., 1997; Phelan et al., 1998). We identified two other murine genes that are highly related to Aop2: Aop2-rs1 and Aop2-rs2, which share $93 \%$ and $80 \%$ nucleotide identity with Aop2, respectively (Phelan et al., 1998). Both related genes lack introns, and are regulated by different promoters. Despite their appearance as pseudogenes, both appear to be expressed in a tissue-specific manner, and Aop2-rs1 may encode a full-length protein. Aop2-rs1 has been identified by reverse transcriptase polymerase chain reaction (RT-PCR) from liver and kidney (Phelan et al., 1998), and expression of Aop2-rs 2 has been demonstrated by RNase protection assay, revealing much lower levels in general, with highest levels in liver (Munz et al., 1997). Therefore, one or both of these genes may encode a functional protein. Another lab claimed to find two Aop2-related intronless genes, which they named CP2 and CP5 (Lee et al., 1999). However, their relationship to Aop2-rs1 and Aop2-rs2 is unclear. First, the authors report only $62 \mathrm{bp}$ of open reading frame sequence for the two genes and the sequences are identical to each other. The evidence that they represent two distinct genes is based on a southern blot, which is not shown. The reported sequence does differ from Aop2$r s 2$, and is identical to Aop2-rs1, but since the sequence is very short and is in a highly conserved region, we cannot determine if it is Aop2-rs1. The isolation of pseudogene-like sequences for other TSA proteins has also been reported, including a highly related pseudogene of PAG (Prosperi et al., 1994), and two recently identified PRx II-like genes (Lim et al., 1998). However, none of these have yet been shown to be expressed. Therefore, the existence of these Aop2-related genes may be physiologically relevant, and their antioxidant activity will need to be measured to determine their functional significance. 


\section{AOP2 structure/function}

To begin to understand the role of AOP2 as a cellular antioxidant, we can examine the activity of other TSA proteins. The original yeast TSA protein was given the name by virtue of the fact that it could protect cellular enzymes from inactivation mediated by metal-catalyzed oxidative systems containing sulfur, but not those containing oxygen (Kim et al., 1988). This was further supported by evidence that yeast TSA uses thiol-containing electron donors to reduce hydroperoxides (Chae et al., 1994a; Kwon et al., 1994; Netto et al., 1996). Several family members have since been shown to possess this activity, and the ability of an enzyme to protect glutamine synthetase from inactivation mediated by such systems has become the standard assay for TSA classification (Chae et al., 1999). Like other TSA proteins, AOP2 has been shown in vitro and in vivo to possess peroxidase activity (Kang et al., 1998a). However, evidence suggests that the endogenous electron donor used by AOP2 may differ. Although all five TSA proteins can reduce $\mathrm{H}_{2} \mathrm{O}_{2}$ in vitro in the presence of DTT, recent studies have shown that AOP2 is the only one that is unable to reduce $\mathrm{H}_{2} \mathrm{O}_{2}$ in the presence of thioredoxin (Fisher et al., 1999; Kang et al., 1998a). This suggests an alternative electron donor for AOP2, which remains unknown.

Since AOP2 may have a novel role as a cellular antioxidant, it is important to elucidate its specific function. However, little is known about its specific substrates or the regions required for its TSA activity. Due to the high degree of evolutionary conservation in amino acid sequence within this family, we can gain some insight into TSA function and specificity by comparison of mammalian TSA proteins (Fig. 1). Alignment of the mammalian consensus sequences reveals three highly conserved motifs. The first motif exhibits striking conservation throughout evolution, and contains the active site cysteine present in all family members from all species. This conserved cysteine residue (corresponding to Cys47 in yeast TSA) has been shown to be the active site of oxidation in a DTT $/ \mathrm{Fe}^{3+} / \mathrm{O}_{2}$ oxidation system (Chae et al., 1994c). Eight other amino acids in this 14 -amino-acid region are $100 \%$ conserved be- tween mammalian TSA proteins, suggesting an essential role in TSA function. A second motif spans 11 amino acids and contains six conserved residues, and the third motif contains 6 conserved residues out of 15 . Although highly conserved, the function of these two motifs is unclear because they are not found in other proteins and are not associated with the active site. In addition to these homologous regions, all TSA proteins except AOP2 have a second conserved cysteine residue, corresponding to Cys170 in yeast TSA. The lack of this second conserved cysteine in AOP2 and its inability to use thioredoxin as an electron donor indicates a unique substrate specificity, and possibly, a novel antioxidant function.

A better understanding of the relationship between the sequence and function of AOP2 can come from analysis of the protein structure. Previous attempts to crystallize native TSA proteins have been complicated by the heterogeneity of intermolecular disulfide interactions (Choi et al., 1998). However, in May of last year the crystal structure of the human AOP2 protein (hORF6) was reported (Choi et al., 1998), the first of the TSA family. The crystallized protein contains two discrete domains and exists as a dimer. The larger amino-terminal domain spanning amino acids 1-174 contains a thioredoxin fold, which encompasses the active cysteine residue. Thioredoxin folds have been found in other antioxidants including glutathione peroxidase and glutathione $S$-transferase and have been shown to be the active site for thioredoxin oxidation (Martin, 1995). The location of the active site cysteine is at the bottom of a narrow pocket and exists as Cys$\mathrm{SOH}$ (cysteine-sulfenic acid) in the crystal. The positioning of two positively charged residues (His 39 and Arg 132) in close proximity (3.76 ̊s) to the active site is thought to lower the $\mathrm{pKa}$ of the thiol by stabilizing its ionic state through hydrogen bonding. Due to the significant distance between two active sites in the dimer, it has been suggested that they would probably function independently (Choi et al., 1998). All three highly conserved TSA motifs are contained within this amino-terminal domain. The second domain (spanning amino acids 175-224) contains five residues that are responsible for dimerization through interactions 
PRXI

PRXII

PRXIII

PRXIV

Aop2

consensus

PRXI

PRXII

PRXIII

PRXIV

AOp 2

consensus

PRXI

PRXII

PRXIII

ERXIV

Aop2

consensus

PRXI

PRXII

PRXIII

PRXIV

Aop2

consensus

PRXI
PRXII
PRXIII
PRXIV
AOp2
consensus

PRXI

FRXII

PPXIII

ERXIV

Aop 2

consensus
MAAAsGRLLhu---SVARXSSSIXhuISAsSLRPSAst. . . . . . RTsLTs.hLhSu Meshs.hLhsSTssptRap+xXXLLLXPLLLFLLXStUlQGhEo--RxRTREPECHFYAG Meshs.hLhss Ts

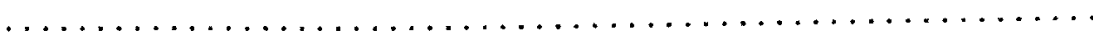

_.................. NSSG . NAKIGaPAPSFKATAVMPOGQEKDISLS-YKGKYVVF - MASG. NAPIGKSAPDEPUTAVVD . GAFKE1KLSDY+GKYVVL ---n-sSQU. KXhFSTSSShHSPAVTQHAPaFKGTAVVN. GEFK-LSLDDFKGKYLVI GQVYPGESSRVSVADHSLHLS. . KAKISKPAPYWEGTAVIS. GEFKELKLTDYRGKYLVE

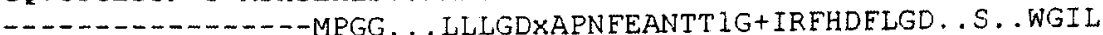

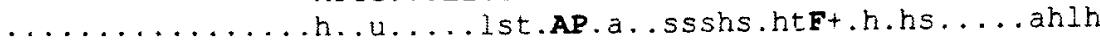

DTYPLDETEVCPTE I IAFSDRA-EFKKINCOVIGASVDSAECHLAN . . INTEKKQGGLG FFYPLDFTIYTCPE I IAFS S +AEDFRKLGCEVLGVSVBSOR SHIAW . . INTPRKEGGLG EFUEIDET EVCETE I VAE SDKANE FHDVNCEVVAVSVDSHESHAAY . . . INT PF KNGGLG

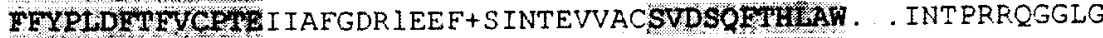
FSHPRD FTPVCWTE LGRAAKLAPE FAKRNVKL IAL STDSYBDIHAYSKD INAYNS St PTE F. aPhDET.vCsTEIhthup.h. - F. phsspliuhSIDS . . SHhAW. . . INs . pppssht

PMNIPLISDEKRT IAQDYGVLK . . AD . . EGISERGLFIIDDKGILPQIT INDLPVGRS PLNI PLLUDVT+PLSPSYGVLK. . . SD . . . EGIAYRGLEI IDUKGVLRQITVNDLPVGRS HMN I SLLSDITKQI SRDYGVLL . . EU . . . UGIALRGLFI IDPNGVIKHLSVNDLPVGRS PIRIPLLSDLSHQISKDYGVYL . . ED. . . SGHTLRGLF I IDDKGIIRQITLNDLPVGRS KLPEPIIDD+\$R-LAILLGMLDPSEKD-PSMPVTARVVEI FGPDKKLKLSILYPATT GPA .h.hsll.D.p..lu..hGhh...t. ...shshRslFlhs.pthL+...1.shssGRs

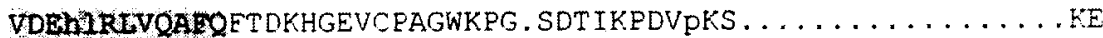

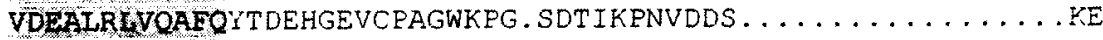
DEESLIRTKAFQ VDETLRTVOAROYTDKHGEVCEAGWKPG.SETI IPDPAGK. . . . . . . . . LK CDE TLRVVXSLQLT UPPXVATPVDWKCG-SVMVI PT I SEEEAKPHFFKGVFTKELESGKK

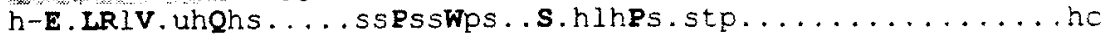
YFSKQK. .
YFSKHN. .
YEPKVPQ.
YFD....
YLFYTEQP
Yh.....

FIG. 1. Amino acid sequence alignment of the TSA family. The human, mouse, and rat homologs for each TSA protein were aligned using ClustalW $(1.7)$ to determine a mammalian consensus sequence for each family member. [The ClustalW default grouping of amino acids issued for amino acid codes: o (alcohol); 1 (aliphatic); a (aromatic); $c$ (charged); h (hydrophobic); $\mathrm{p}$ (polar); s (small); u (tiny); t (turnlike); - (negative); + (positive).]. These consensus sequences were aligned and a consensus sequence for the entire TSA family was generated using the Consensus program. Amino acid identities are shown in uppercase boldface in the consensus sequence.

with residues surrounding the active site of the other subunit. These include Pro191, which resides in the entrance to the active site of the opposing monomer. While the intramolecular contacts appear to be minimal for dimerization, these intermolecular bonds allow for close proximity between domain I of one monomer and domain II of the other. Dimerization of other TSA proteins has also been demonstrated. Yeast TSA exists as a homodimer, and the oxidized Cys 47 reacts rapidly with the carboxy-terminal cysteine (\#170) (conserved in 2Cys TSA proteins) to form a disulfide bond, which subsequently can be reduced by interaction with the redox active cysteines of thioredoxin (Chae et al., 1994c). This type of interac- 
tion has also been shown for other 2-Cys peroxiredoxins, suggesting a common mechanism for peroxidase function. However, the absence of the second conserved cysteine in the AOP2 dimer suggests the importance of other residues in mediating its activity, and raises the possibility that the distinct dimer-stabilization mechanism specifies a unique peroxidase activity.

In an effort to elucidate its unique function further, all identified AOP2 protein sequences from a range of different organisms were compared. The human AOP2 sequence, with evolutionarily conserved residues, is shown in Fig. 2. Forty-eight amino acids in the AOP2 protein have been conserved throughout evolution, $21 \%$ of the entire protein. Half of these are also conserved among the other TSA family members. The motif containing the active site cysteine, which shows the highest identity among TSA proteins, is also most highly conserved between AOP2 proteins from divergent species ( 13 out of 14 residues). This is in contrast to motif 2 , which contains 4 conserved residues out of 11 , and motif 3 , which contains 6 conserved residues out of 15 . Other amino acids outside of these motifs are also conserved throughout the entire family, including Arg132 and Pro191, which have both been localized around the active site pocket of the AOP2 dimer (Choi et al., 1998). Interestingly, there are also amino acids that are unique to the AOP2 lineage, yet conserved in other TSA family members, suggesting some role in AOP2 specificity. These in- clude His 39 and Thr48, both of which are located in the first highly conserved motif. His39, replaced by either tyrosine or tryptophan in the thioredoxin-peroxiredoxins, was shown to be critical for establishing a positively charged environment surrounding the active site for cysteine oxidation (Choi et al., 1998). Thr48, which corresponds to a conserved proline in all other family members, is adjacent to the active cysteine and is important in dimer stabilization (Choi et al., 1998). Other AOP2 amino acids that are nonvariant between prokaryotes and eukaryotes, yet different in the other family members, are Asn156 and Leu167 in motif 3, and Lys199 and Phe202 within the AOP2-specific carboxy-terminal region. Analysis of the sequence also exposes the absence of a secretion signal suggesting an intracellular function for the protein, which is consistent with the subcellular localization. Finally, it is worth noting the presence of putative post-translational modification sites, including four PKC phosphorylation sites, three $\mathrm{CK}-2$ phosphorylation sites, and three potential $N$-myristylation sites within the human protein (see Fig. 2). At least two putative PKC phosphorylation sites involve residues that are unique to $A O P 2$, and may be important in the regulation of AOP2 activity.

Comparison of the structure and function of AOP2 creates many possibilities about the unique function of AOP2. The amino-terminal domain likely includes those residues that are critical for peroxidase activity. The conser-

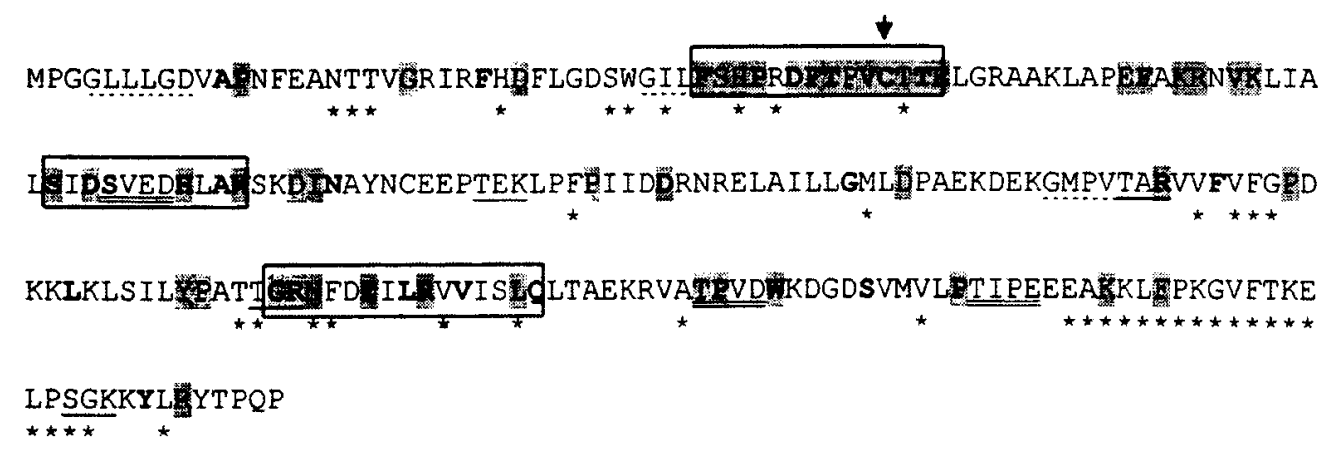

FIG. 2. Human AOP2 amino acid sequence and evolutionarily conserved residues. The boxes designate conserved motifs between the TSA family. Amino acids in boldface are $100 \%$ conserved between all mammalian TSA proteins. Amino acids that show $100 \%$ identity between Aop2 homologs are shaded, and the active site cysteine common to all TSA proteins is indicated with an arrow. Amino acids denoted with an asterisk are unique to AOP2, yet conserved between all other mammalian TSA proteins. Putative sites for (PKC) phosphorylation (underlined), CK2 phosphorylation (double underlined), and $N$-myristolylation (dotted underlined) are indicated. 
vation of Arg132 and Pro191 in all TSA proteins suggests basic roles in dimer stabilization and active site configuration. Whereas Arg132 likely plays a basic role in dimerization, Pro191, which is localized near the entrance to the active site pocket of the other monomer, may be an important factor in regulating access to the active site in all TSA proteins. Although the active site of AOP2 may possess a thiol-specific antioxidant activity similar to that of other family members, it may specify a different electron donor due to specific residues within and surrounding this domain. The striking conservation of His39 and Thr48 between divergent species may suggest its importance in the specificity of AOP2 for particular electron donors, since these residues are uniquely conserved in AOP2. It has also been suggested that the role of His 39 in the dimer may mimic that of Cys 170 in 2-Cys proteins (Choi et al., 1998). The substitution of threonine for proline at residue 48 may instead be critical in substrate specificity due to its nearby contacts within the dimer. This may also be true for the specific amino acids found in motif 3 , which differ considerably from the other TSA proteins. In contrast, the internal highly conserved motif shows little uniqueness in AOP2 proteins, and thus, may mediate a function common to all TSA proteins, although its significance is unclear. Because this family is capable of forming heterodimers as well as homodimers, this interaction may also influence substrate specificity. It is also important to note that 18 amino acids near the carboxyl terminus of AOP2 show no homology with other TSA family members. Interestingly, this region does share limited homology with a portion of the cofactor-binding site of porphobilinogen deaminase proteins (Q57989), all of which possess the following motif: $K G(V / L) F(T / V) K E(L / I)$. However, the specific function of this region is unknown.

Despite the demonstrated thiol-specific antioxidant activity of AOP2, and its obvious sequence homology with the TSA family, other functions for AOP2 have been suggested. The Aop 2 cDNA was also isolated from human (Frank et al., 1997), and then mouse (Munz et al., 1997), as a glutathione peroxidase (GPx) for its high amino-terminal amino acid homology with a bovine nonselenium glutathione perox- idase, of which only the amino-terminal 24 amino acids were known. While the human protein demonstrates no glutathione peroxidase activity as measured by its inability to protect glutamine synthetase from inactivation in the presence of glutathione (Kang et al., 1998a), bovine AOP2 was able to use glutathione as the electron donor in experiments measuring NADPH consumption (Fisher et al., 1999). Although these results may reflect a species difference in function, it is unlikely due to their $98 \%$ sequence similarity. Thus, the in vivo electron donor is still unknown. Furthermore, the question as to what is the best assay for AOP2 activity is raised. This is reiterated by the demonstration that AOP2 has calcium-independent phospholipase activity with maximal activity at $\mathrm{pH} 4$, as determined by measured enzyme activity in vitro after enzyme purification (Kim et al., 1997, 1998). The presence of a lipase motif (GXSXG) in the amino-terminal portion of the mammalian AOP2 proteins (Kim et al., 1997) was an additional piece of suggestive evidence for this activity. A low level of phospholipase activity has, in fact, been reported by another group (Kang et al., 1998a), but it may be orders of magnitude less than its peroxidase activity and does not require the GXSXG motif. In addition, the acid-dependent nature of this lipase activity means it is probably not functional in the neutral $\mathrm{pH}$ of the cytosol. Although AOP2 shows little sequence homology with the few members of the calcium-independent PLA $A_{2}$ family that have been cloned and sequenced, the fact that phospholipase activity has been demonstrated by independent investigations may reveal a dual function for this protein. On the other hand, the observation that this activity is significantly less than its antioxidant activity may instead simply reflect a specificity of AOP2 for phospholipids. Consistent with this idea, a recent study has shown that phospholipid hydroperoxides can act as substrates for recombinant bovine and human AOP2 expressed and purified from E. coli (Fisher et al., 1999). In contrast, glutathione peroxidase could not reduce phospholipid hydroperoxides. Maximal enzymatic activity for AOP2 was shown to occur between $\mathrm{pH} 7$ and 8, concordant with the cytosolic localization of AOP2. Nevertheless, the ability of 
AOP2 to reduce PLPC hydroperoxides, and its phospholipase activity, presents a unique potential function for a cellular antioxidant.

Thus, the true in vivo function of AOP2 remains to be determined. Given the above-described evidence, three models for AOP2 activity can be postulated. These are shown in Fig. 3, A-C. It is known that AOP2 can exist in a homodimeric form. Thus, model A proposes that AOP2 exists as a redox-sensitive dimer in the cytosol, which can be changed from a reduced to oxidized state without additional modification. In this scenario, the mechanism of AOP2 regulation might be at the level of de novo transcription/translation. This is supported by evidence that Aop 2 transcription is induced in response to KGF (Frank et al., 1997). However, evidence from other TSA proteins suggests that it may also participate in hetero- dimeric interactions, and may exist in monomeric form under some circumstances. Thus, model $\mathrm{B}$ postulates that AOP2 may be reduced in its monomeric state, and can form either a homo- or heterodimer upon activation by an unidentified signal. In this model, the activation event may be phosphorylation mediated by signaling from the external stimulus, which could result in a conformational change in the protein. Dimerization may, in turn, expose the active cysteine to be oxidized by some hydroperoxide, ultimately leading to dissociation of the complex and reduction of the monomers by the thiol-containing electron donor. In contrast, model $\mathrm{C}$ postulates that oxidation of the AOP2 monomers by $\mathrm{H}_{2} \mathrm{O}_{2}$ might lead to its dimerization, and subsequent reduction by the unknown electron donor. While the actual mechanism of AOP2 activity remains to be de-

A.

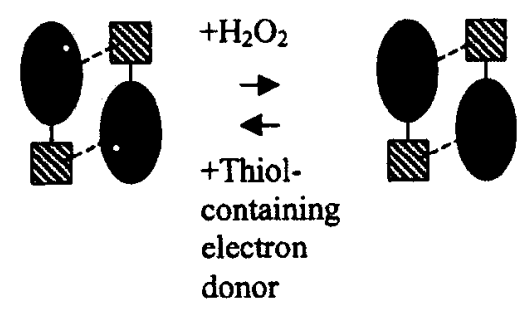

B.
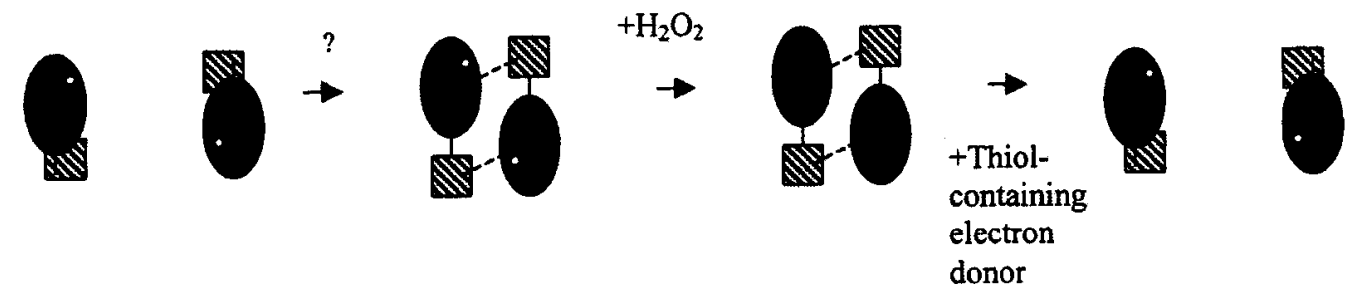

electron

C.
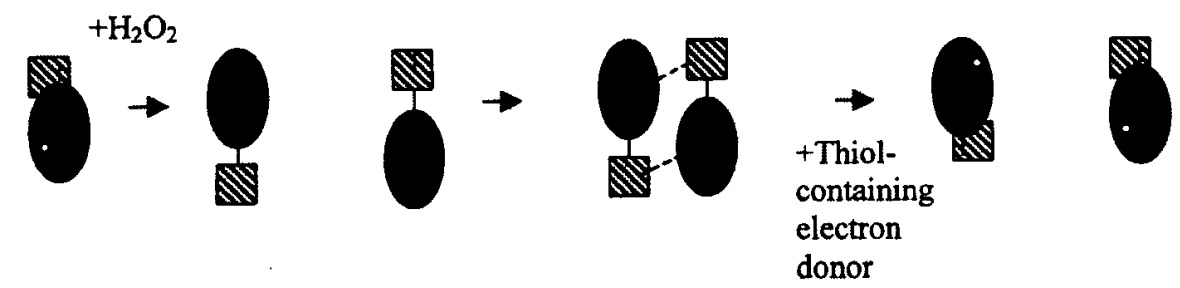

FIG. 3. Models for AOP2 activity modulation. The purple region indicates domain I and the blue region indicates domain II. The active site cysteine is indicated by the small circle in domain I, which can exist in a reduced state (white) or an oxidized state (black). A: AOP2 exists as a dimer in the cytosol, and its activity is modulated by the redox-state of the protein. B. AOP2 exists in an inactive monomeric state in the cytosol and, upon stimulation by a specific signal (i.e., phosphorylation), forms a dimer. The active site cysteines of the dimer can then be oxidized, leading to dissociation of the complex, and subsequent reduction of the active site. C. AOP2 is oxidized in its monomeric form in the cytosol, leading to dimerization. Formation of the dimer allows for reduction to occur and subsequent disassembly of the complex. 
termined, significant progress will come from identification of the in vivo electron donor and the hydroperoxide substrate specificity. For example, the abundance of the electron donor and the nature of the hydroperoxide may specify AOP2 activity. Furthermore, it is important to remember that the two highly related genes Aop2-rs1 and Aop2-rs2 may encode proteins with similar activity as AOP2. In fact, a 15amino-acid region containing the active site cysteine residue is conserved between all three genes (Phelan et al., 1998), including His39, which is conserved in all 1-Cys peroxiredoxin proteins. Because these amino acids are thought to create and stabilize the active site of the enzyme, these related proteins may also be important in the cellular stress response.

\section{Potential role for $A O P 2$ in cell signaling and pathogenesis}

Due to the known involvement of redox proteins in cell communication, several labs are currently investigating the function of the TSA family in signal transduction. Many studies have reported the generation of ROS during cytokine and growth factor action, hormone secretion, ion transport, gene transcription, and apoptosis (Lander, 1997). Evidence suggests that these stimuli use reactive intermediates as mediators of intracellular signals. Although the mechanism of action for this group of messengers is unclear, one suggestion has been the inactivation of protein tyrosine phosphatases by ROS, thereby allowing necessary protein phosphorylation during transduction of a signal (Sundaresan et al., 1995). This has been shown for transmission of the platelet-derived growth factor (PDGF) signal by $\mathrm{H}_{2} \mathrm{O}_{2}$. While the role of ROS in such pathways is becoming increasingly supported, the role of antioxidant proteins as regulators of such messengers has just recently been proposed. The function of antioxidants as ROS scavengers may result in sequestering of ROS and subsequent suppression of phosphatase inactivation, leading to signal termination. Thiol-specific antioxidants may have a specific role in this process by sequestering specific ROS only in the presence of a thiol-containing electron donor generated by specific signals. Thiol-specific antioxidants have, in fact, been shown to mediate certain responses to growth factors. Overexpression of PRx I and Prx II in cultured cells resulted in the elimination of intracellular $\mathrm{H}_{2} \mathrm{O}_{2}$ induced by growth factor stimulation (Kang et al., 1998b). PRx II was also able to block NF- $\kappa$ B activation by $\mathrm{H}_{2} \mathrm{O}_{2}$ or tumor necrosis factor- $\alpha$ (TNF- $\alpha$ ), suggesting an important role in cell signaling by sequestering hydroperoxides. Furthermore, antioxidant enzyme 372 (AOE372) was able to inhibit NF- $\kappa$ B activation by regulating $I-\kappa \mathrm{B}$ phosphorylation in the cytoplasm (Jin et al., 1997). This is consistent with the idea that antioxidants can participate in signaling by sequestering hydroperoxides, thereby relieving suppression of critical phosphatases. There is growing evidence that AOP2 is also involved in these processes. First, the human Aop 2 was cloned (as KRG-1) by one lab on the basis of its super-induction in keratinocytes by keratinocyte growth factor (KGF) (Frank et al., 1997). Munz et al. went on to demonstrate over a 100-fold increase in murine Aop 2 expression during the first $24 \mathrm{hr}$ of cutaneous would repair, when KGF levels are very significantly elevated (Munz et al., 1997). This effect was specific for KGF, and was localized to the hyperproliferative epithelial edge, suggesting a role in cell proliferation. We have also shown that Aop2 expression is induced in response to oxidative stress in a mouse hepatocyte cell line (data not shown). More directly, Kang et al. have shown that while PDGF stimulation of NIH-3T3 cells results in elevated intracellular $\mathrm{H}_{2} \mathrm{O}_{2}$, overexpression of Aop 2 inhibits this increase, and this inhibition depends on the active site Cys47 (Kang et al., 1998b). Thus, the unique specificity of AOP2 for particular cellular substrates may allow for the transmission of a defined set of signals through sequestering of specific hydroperoxides.

A model for the role of AOP2 in cellular stress response and signal transduction is shown in Fig. 4. It is clear that $\mathrm{H}_{2} \mathrm{O}_{2}$ can be induced in response to a wide variety of stimuli, including growth factors, cytokines, and hormones, all of which utilize similar signaling pathways. Although specific kinases are activated in response to these stimuli, $\mathrm{H}_{2} \mathrm{O}_{2}$ accumulation may lead to the inactivation of protein tyrosine phosphatases. This event would 


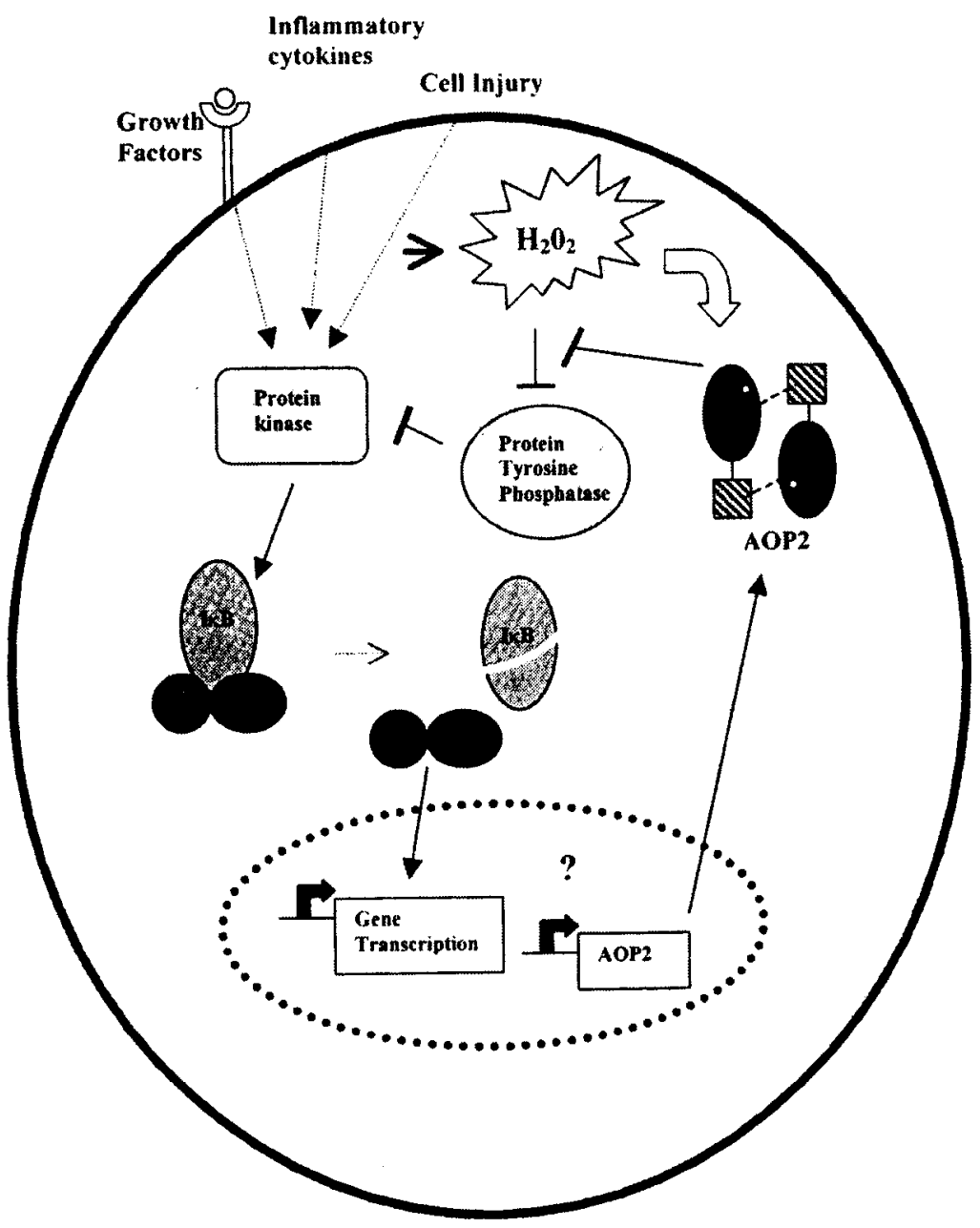

FIG. 4. Hypothetical model for AOP2 action in signal transduction. Extracellular signals such as growth factors and cytokines activated specific protein kinases in the cell that lead to activation of transcription factors such as NF$\kappa \mathrm{B}$. Generation of $\mathrm{H}_{2} \mathrm{O}_{2}$ by these signals results in the inactivation of protein tyrosine phosphatases, allowing transmission of the signal. Transcription of AOP2 is induced by an unknown mechanism, and AOP2 protein sequesters $\mathrm{H}_{2} \mathrm{O}_{2}$ by an unknown electron donor, inhibiting phosphatase inactivation and facilitating regulation of the signal.

allow for propagation of the signal via phosphorylation of critical factors such as NF- $\kappa$ B, leading to specific gene transcription. Because thioredoxin-dependent antioxidant defense systems have shown greatest selectivity for NF$\kappa \mathrm{B}$ activation, this pathway may likely serve as a target for AOP2 regulation. We know that Aop2 expression is highly induced in keratinocytes in response to KGF. We also know that Aop 2 overexpression leads to reduction in the intracellular pool of $\mathrm{H}_{2} \mathrm{O}_{2}$ generated by PDGF stimulation. Thus, some unknown mediator may lead to induction of Aop2 expression (and/or activation of existing AOP2 protein) resulting in the accumulation of $A O P 2$ in the cytosol and the sequestering of $\mathrm{H}_{2} \mathrm{O}_{2}$. The presence of multiple heat shock elements and
Sp1 binding sites in the proximal promoter suggest that these factors may be involved in its induction. This reduction in $\mathrm{H}_{2} \mathrm{O}_{2}$ may inhibit phosphatase inactivation, and allow for regulation of the signal. Although speculative at this point, this model proposes an efficient method for reduction of hydroperoxides and regulation of intracellular signals by AOP2.

As more antioxidants have emerged over the past decade, there have been many reports linking them with pathogenesis. Diseases ranging from diabetes to cancer and heart disease are associated in some way with the production of free radicals and the involvement of inborn defense mechanisms. Recent studies have implicated TSA proteins in such pathophysiological processes. Studies have proposed a role 
for TSA proteins in inhibition of apoptosis, as shown by the ability of stably transfected human TPx 11 to inhibit apoptosis induced by chemicals or serum deprivation (Zhang et al., 1997). TPx II overexpression was found to inhibit lipid peroxidation and $\mathrm{H}_{2} \mathrm{O}_{2}$ accumulation in cells, suggesting a role upstream of Bcl2 in apoptosis suppression (Zhang, et al., 1997). Furthermore, expression of mouse TPx was able to protect neuronal cells from apoptosis in the absence of nerve growth factor (NGF) and serum, suggesting a similar role (Ichimiya et al., 1997).

There is some evidence to suggest that Aop2 plays a role in certain disease processes, as well. Frank et al. have shown significantly elevated levels of the human gene in psoriasis-affected skin (Frank et al., 1997). This condition is an inflammatory disorder and involves elevations in cellular stress responses genes. Thus, overexpression of Aop 2 in these cells may provide added protection in the face of increased oxidative stress. We also have evidence to suggest that AOP2 may play a role in atherosclerosis resistance in mice. Genetic studies from our laboratory have identified Aop 2 as a candidate for a quantitative trait locus (QTL) underlying an atherosclerosis susceptibility difference between certain inbred strains of mice. This locus, originally named $A t h 1$, is responsible for resistance to high-density lipoprotein (HDL) reduction and atherosclerosis in $\mathrm{C} 3 \mathrm{H} /$ $\mathrm{HeJ}$ and BALB/cJ mice fed a high-fat diet, as compared to susceptible C57BL/6J mice (Paigen et al., 1987). Our evidence for involvement of AOP2 in atherosclerosis includes colocalization of Aop2 to the same $0.05 \mathrm{cM}$ interval as Ath1, and differences in amino acid sequence and mRNA expression of Aop 2 between resistant and susceptible strains (Beier et al., in preparation). In addition, the role of lipid oxidation in atherosclerosis progression has been well established, implicating a role for antioxidant proteins in this process. Thus, AOP2 may play a role in the liver by reducing exposure of lipoproteins to oxidative modification, or it may protect cells in the artery wall from oxidative damage initiated by low-density lipoprotein (LDL) oxidation. The presence of putative binding sites for heat shock factor (HSF), implicated in atherosclerosis, and sterol response element binding protein (SREBP), a known regulator of lipid metabolism, in the Aop2 promoter suggests possible transcriptional regulation of Aop 2 in these processes. We are now testing the role of AOP2 in atherosclerosis by a transgenic rescue experiment. Therefore, mounting evidence suggests that AOP2 is likely an important cellular antioxidant that may play a role in basic biochemical defense mechanisms as well as clinical disease. Further investigation of the substrate specificity for this protein, and identification of its in vivo electron donor will help to uncover its actual function and specificity. In addition, we hope to elucidate the pathways that lead to transcriptional regulation of Aop2 and modulation of its activity. Together, significant progress can be made in understanding the role of this novel antioxidant in cellular stress response and signal transduction.

\section{ACKNOWLEDGMENTS}

The communicated work was performed in the laboratory of Beverly Paigen at The Jackson Laboratory, Bar Harbor, ME: The work was supported by ASTRA Pharmaceuticals and NHLBI 1-F32-HL10124-01. I would like to thank Mathew A. Merrill for excellent technical assistance, and Beverly Paigen for support.

\section{ABBREVIATIONS}

aiPLA2, acidic calcium-independent phospholipase $\mathrm{A}_{2} ; \mathrm{AOE} 372$, antioxidant enzyme 372; Aop2, antioxidant protein 2; Aop2-rs, antioxidant protein 2-related sequence; Ath, atherosclerosis susceptibility locus; $\mathrm{cM}$, centimorgan; $C K$, casein kinase; $C P$, genomic fragment of 1-Cys peroxiredoxin; DTT, diothiothreitol; EST, expressed sequence tag; GPx, glutathione peroxidase; $\mathrm{H}_{2} \mathrm{O}_{2}$, hydrogen peroxide; HBP, heme binding protein; HDL, high-density lipoprotein; HO', hydroxyl radical; hORF, human open reading frame; HSF, heat shock factor; IVH, influenza virus hemagglutinin; KGF, keratinocyte growth factor; KRG, keratinocyteregulated gene; LDL, low-density lipoprotein; MGD, mouse genome database; MSP, macro- 
phage stress protein; NF- $\kappa \mathrm{B}$ (nuclear factor kappa B; NGF, nerve growth factor; NKEF, natural killer cell enhancing factor; $\mathrm{NO}$, nitric oxide; $\mathrm{O}_{2}{ }^{-}$, superoxide anion; OSF, osteoblastspecific factor; PAG, proliferation-associated gene; PDGF, platelet-derived growth factor; PKC, protein kinase $C$; PLA $_{2}$, phospholipase $\mathrm{A}_{2} ; \mathrm{PRx}$, peroxiredoxin; $\mathrm{QTL}$, quantitative trait locus; ROS, reactive oxygen species; RT-PCR, reverse transcriptase polymerase, chain reaction; SREBP, sterol response element binding protein; TDPX, thioredoxin peroxidase; TDX, thioredoxin peroxidase; TDXM, mitochondrial thioredoxin peroxidase; TNF- $\alpha$, tumor necrosis factor- $\alpha$; TPx, thioredoxin-dependent peroxide reductase; TSA, thiol-specific antioxidant.

\section{REFERENCES}

ANDREEVA, S.G., MERKULOVA, M.I., SHUVAEVA, T.M., NOVOSELOV, V.I., PESHENKO, I.V., NOVOSELOV, S.M., FESENKO, E.E., and LIPKIN, V.M. (1998). Cloning and sequencing of the secretory $28-\mathrm{kDa}$ protein from rat olfactory epithelium. Bioorg. Khim. 24, 816-821.

BEIER, D.R., PHELAN, S.A., MERRILL, M.A., and PAIGEN, B. (1999). The role of Aop2 (antioxidant protein 2) in atherosclerosis resistance in mice. (manuscript in preparation)

CHAE, H.Z., KIM, I.H., KIM, K., and RHEE, S.G. (1993). Cloning, sequencing, and mutation of thiol-specific antioxidant gene of Saccharomyces cerevisiae. J. Biol. Chem. 268, 16815-16821.

CHAE, H.Z., CHUNG, S.J., and RHEE, S.G. (1994a). Thioredoxin-dependent peroxide reductase from yeast. J. Biol. Chem. 269, 27670-27678.

CHAE, H.Z., ROBINSON, K., POOLE, L.B., CHURCH, G., STORZ, G., and RHEE, S.G. (1994b). Cloning and sequencing of thiol-specific antioxidant from mammalian brain: alkyl hydroperoxide reductase and thiol-specific antioxidant define a large family of antioxidant enzymes. Proc. Natl. Acad Sci. USA 91, 7017-7021.

CHAE, H.Z., UHM, T.B., and RHEE, S.G. (1994c). Dimerization of thiol-specific antioxidant and the essential role of cysteine 47. Proc. Natl. Acad. Sci. USA 91, 7022-7026.

CHAE, H.Z., KANG, S.W., and RHEE, S.G. (1999). Isoforms of mammalian peroxiredoxin that reduce peroxides in presence of thioredoxin. Methods Enzymol. 300, 219-226.

CHOI, H.-J., KANG, S.W., YANG, C.-H., RHEE, S.G., and RYU, S.-E. (1998). Crystal structure of a novel human peroxidase enzyme at $2.0 \mathrm{~A}$ resolution. Nature Struct. Biol. 5, 400-406.

FISHER, A.B., DODIA, C., MANEVICH, Y., CHEN, J.W., and FEINSTEIN, S.I. (1999). Phospholipid hydroperox- ides are substrates for non-selenium glutathione peroxidase. J. Biol. Chem. 23, 21326-21334.

FRANK, S., MUNZ, B., and WERNER, S. (1997). The human homologue of a bovine non-selenium glutathione peroxidase is a novel keratinocyte growth factor-regulated gene. Oncogene 14, 915-921.

HARIDAS, V., NI, J., MEAGER, A., SU, J., YU, G.L., ZHAI, Y., KYAW, H., AKAMA, K.T., HU, J., VAN, E.L., and AGGARWAL, B.B. (1998). TRANK, a novel cytokine that activates NF-kappa B and c-Jun N-terminal kinase. J. Immunol. 161, 1-6.

IAKOUBOVA, O.A., PACELLA, L.A., HER, H., and BEIER, D.R. (1997). LTW4 protein on mouse chromosome 1 is a member of a family of antioxidant proteins. Genomics 42, 474-478.

ICHIMIYA, A.S., DAVIS, J.G., O'ROURKE, D.M., KATSUMATA, M., and GREENE, M.I. (1997). Murine thioredoxin peroxidase delays neuronal apoptosis and is expressed in areas of the brain most susceptible to hypoxic and ischemic injury. DNA Cell Biol. 16, 311-321.

ISHII, T., YAMADA, M., SATO, H., MATSUE, M., TAKETANI, S., NAKAYAMA, K., SUGITA, Y., and BANNAl, S. (1993). Cloning and characterization of a 23-kDa stress-induced mouse peritoneal macrophage protein. J. Biol. Chem. 268, 18633-18636.

IWAHARA, S., SATOH, H., SONG, D.X., WEBB, J., BURLINGAME, A.L., NAGAE, $Y$., and MULLEREBERHARD, U. (1995). Purification, characterization, and cloning of a heme-binding protein $(23 \mathrm{kDa})$ in rat liver cytosol. Biochemistry 34, 13398-13406.

JIN, D.-Y., CHAE, H.Z., RHEE, S.G., and JEANG, H.-T. (1997). Regulatory role for a novel human thioredoxin peroxidase in NF- $\kappa$ B activation. J. Biol. Chem. 268, 18633-18636.

KANG, S.W., BAINES, I.C., and RHEE, S.G. (1998a). Characterization of a mammalian peroxiredoxin that contains one conserved cysteine. J. Biol. Chem. 273, 6303-6311.

KANG, S.W., CHAE, H.Z., SEO, M.S., KIM, K.H., BAINES, I.C., and RHEE, S.G. (1998b). Mammalian peroxiredoxin isoforms can reduce hydrogen peroxide generated in response to growth factors and tumor necrosis factor-alpha. J. Biol. Chem. 273, 6297-6302.

KAWAI, S., TAKESHITA, S., OKAZAKI, M., KIJUNO, R., KUDO, A., and AMANN, E. (1994). Cloning and characterization of OSF-3, a new member of the MER5 family, expressed in mouse osteoblastic cells. J. Biochem. 115, 641-643.

KIM, K., KIM, I.H., LEE, K.Y., and RHEE, S.G. (1988). The isolation and purification of a specific "protector" protein which inhibits enzyme inactivation by a thiol/ $\mathrm{Fe}(\mathrm{III}) / 02$ mixed-function oxidation system. J. Biol. Chem. 263, 4704-4711.

KIM, T.-S., SUNDARESH, C.S., FEINSTEIN, S.I., DODIA, C., SKACH, W.R., JAIN, M.K., NAGASE, T., SEKI, N., ISHIKAWA, K., NOMURA, N., and FISHER, A.B. (1997). Identification of a human cDNA clone for lysosomal type $\mathrm{Ca}^{2+}$-independent phospholipase $\mathrm{A}_{2}$ and properties of the expressed protein. J. Biol. Chem. 272, 2542-2550. 
KIM, T.-S., DODIA, C., CHEN, X., HENNIGAN, B.B., JAIN, M., FEINSTEIN, SI, and FISHER, A.B. (1998). Cloning and expression of rat lung acidic $\mathrm{Ca}^{2+}$-independent $\mathrm{PLA}_{2}$ and its organ distribution. Am. J. Physiol. 274, L750-L761.

KWON, S.J., PARK, J.W., and KIM, K. (1994). Inhibition of metal-catalyzed oxidation systems by a yeast protector protein in the presence of thiol. Biochem. Mol. Biol. Int. 32, 419-427.

LANDER, H.M. (1997). An essential role for free radicals and derived species in signal transduction. FASEB. J. 11, 118-124.

LEE, T-H, YU, S.-L., KIM, S.-U., KIM, Y.-M., CHOI, I., KANG, S.-W., RHEE, S.G., and YU, D.-Y. (1999). Characterization of the murine gene encoding 1-Cys peroxiredoxin and identification of highly homologous genes. Gene 234, 337-344.

LIM, M.J., CHAE, H.Z., RHEE, S.G., YU, D.-Y., LEE, K.-K., and YEOM, Y.I. (1998). The type II peroxiredoxin gene family of the mouse: molecular structure, expression and evolution. Gene 216, 197-205.

LIM, Y.S., CHA, M.K., KIM, H.K., and KIM, I.H. (1994). The thiol-specific antioxidant protein from human brain: gene cloning and analysis of conserved cysteine regions. Gene 140, 279-284.

MARTIN, J.L. (1995). Thioredoxin-a fold for all reasons. Structure 3, 245-250.

MATSUMOTO, A.L., OKADO, A., FIJII, T., FUJII, J., EGASHIRA, M., NIIKAWA, N., and TANIGUCHl, N. (1999). Cloning of the peroxiredoxin gene family in rats and characterization of the fourth member. Febs Lett. 443, 246-250.

MUNZ, B., FRANK, S., HUBNER, G., OLSEN, E., and WERNER, S. (1997). A novel type of glutathione peroxidase: expression and regulation during wound repair. Biochem. J. 326, 579-585.

NETTO, L.E.S., CHAE, H.Z., KANG, S.W., RHEE, S.G., and STADTMAN, E.R. (1996). Removal of hydrogen peroxide by thiol-specific antioxidant enzyme (TSA) is involved with its antioxidant properties. TSA possesses thiol peroxidase activity. J. Biol. Chem. 271, 15315-15321.

PAIGEN, B., MITCHELL, D., REU, K., MORROW, A., LUSIS, A.J., and LEBOEUF, R.C. (1987). Ath-1, a gene determining atherosclerosis susceptibility and high density lipoprotein levels in mice. Proc. Natl. Acad. Sci. USA 84, 3763-3767.

PESHENKO, I.V., NOVOSELOV, V.I., EVDOKIMOV, V.A., NIKOLAEV, Y.V., SHUVAEVA, T.M., LIPKIN, V.M., and FESENKO, E.E. (1996). Novel 28-kDa secretory protein from rat olfactory epithelium. FEBS Lett. 381, 12-14.
PHELAN, S.A., JOHNSON, K.A., BEIER, D.R., and PAIGEN, B. (1998). Characterization of the murine gene encoding Aop2 (antioxidant protein 2) and identification of two highly related genes. Genomics 54, 132-139.

PROSPERI, M.-T., FERBUS, D., KARCZINSKI, I., and GOUBIN, G. (1993). A human cDNA corresponding to a gene overexpressed during cell proliferation encodes a product sharing homology with amoebic and bacterial proteins. J. Biol. Chem. 268, 11050-11056.

PROSPERI, M.-T., APIOU, F., DUTRILLAUX, B., and GOUBIN, G. (1994). Organization and chromosomal assignment of two human PAG gene loci: PAGA encoding a functional gene and PAGB a processed pseudogene. Genomics 19, 236-241.

RHEE, S.G., and CHAE, H.Z. (1994). Thioredoxin peroxidase and peroxiredoxin family. Mol. Cells. 4, 137-142.

SCHERENS, B., EL BAKKOURY, M., VIERENDEELS, F., DUBOIS, E., and MESSENGUY, F. (1993). Sequencing and functional analysis of a $32,560 \mathrm{bp}$ segment on the left arm of yeast chromosome II. Identification of 26 open reading frames, including the KIP1 and SEC17 genes Yeast 9, 1355-1371.

SHAU, H., BUTTERFIELD, L.H., CHIU, R., and KIM, A. (1994). Cloning and sequence analysis of candidate human natural killer-enhancing factor genes. Immunogenetics 40, 129-134.

SUNDARESAN, M., YU, Z.-S., FERRANS, V.J., IRANI, K., and FINKEL, T. (1995). Requirement for generation of $\mathrm{H}_{2} \mathrm{O}_{2}$ for platelet-derived growth factor signal transduction. Science 270, 296-299.

TSUJI, K., COPELAND, N.G., JENKINS, N.A., and OBINATA, M. (1995). Mammalian antioxidant protein complements alkylhydroperoxide reductase (ahpC) mutation in Escherichia coli. Biochem. J. 307, 377-381.

YAMAMOTO, T., MATSUI, Y., NATORI, S., and OBINATA, M. (1989). Cloning of a housekeeping-type gene (MER5) preferentially expressed in murine erythroleukemia cells. Gene 80, 337-343.

ZHANG, P., LIU, B., KANG, S.W., SEO, M.S., RHEE, S.G., and OBEID, L.M. (1997). Thioredoxin peroxidase is a novel inhibitor of apoptosis with a mechanism distinct from that of Bcl-2. J BIol. Chem. 272, 30615-30618.

Address reprint requests to: Dr. Shelley A. Phelan Department of Biology Fairfield University Fairfield, Connecticut 06430

E-mail: sphelan@Fair1.Fairfield.edu 


\section{This article has been cited by:}

1. Aron B. Fisher . 2011. Peroxiredoxin 6: A Bifunctional Enzyme with Glutathione Peroxidase and Phospholipase A2 Activities. Antioxidants \& Redox Signaling 15:3, 831-844. [Abstract] [Full Text] [PDF] [PDF Plus]

2. Yi-Min ZHU, Qi XU, Lai-Rong DONG, Zhi-Ming LIU. 2011. Identification of Proteins Associated With let-7a in Gastric Carcinoma Cell Line SGC-7901 by Proteomics*. PROGRESS IN BIOCHEMISTRY AND BIOPHYSICS 38:5, 441-448. [CrossRef]

3. Y. Hirota, N. Acar, S. Tranguch, K. E. Burnum, H. Xie, A. Kodama, Y. Osuga, I. Ustunel, D. B. Friedman, R. M. Caprioli, T. Daikoku, S. K. Dey. 2010. Uterine FK506-binding protein 52 (FKBP52)-peroxiredoxin-6 (PRDX6) signaling protects pregnancy from overt oxidative stress. Proceedings of the National Academy of Sciences 107:35, 15577-15582. [CrossRef]

4. Ying Ding, Bin Lu, Dongbo Chen, Lina Meng, Yan Shen, Shuo Chen. 2010. Proteomic analysis of colonic mucosa in a rat model of irritable bowel syndrome. PROTEOMICS 10:14, 2620-2630. [CrossRef]

5. T. M. Shuvaeva, V. I. Novoselov, E. E. Fesenko, V. M. Lipkin. 2009. Peroxiredoxins, a new family of antioxidant proteins. Russian Journal of Bioorganic Chemistry 35:5, 523-537. [CrossRef]

6. Bridget M. Gallagher, Shelley A. Phelan. 2007. Investigating transcriptional regulation of Prdx6 in mouse liver cells. Free Radical Biology and Medicine 42:8, 1270-1277. [CrossRef]

7. Flavia Merigo, Donatella Benati, Marzia Chio, Francesco Osculati, Andrea Sbarbati. 2006. Secretory cells of the airway express molecules of the chemoreceptive cascade. Cell and Tissue Research 327:2, 231-247. [CrossRef]

8. Marina Simeone, Shelley A. Phelan. 2005. Transcripts associated with Prdx6 (Peroxiredoxin 6) and related genes in mouse. Mammalian Genome 16:2, 103-111. [CrossRef]

9. P. Ye, M. Simonian, M. A. Nadkarni, A. A. DeCarlo, C. C. Chapple, N. Hunter. 2005. Identification of epithelial auto-antigens associated with periodontal disease. Clinical and Experimental Immunology 139:2, 328-337. [CrossRef]

10. Seong Jin Yu, Ju Ran Kim, Chae Kwan Lee, Jeong Eun Han, Jae Hyun Lee, Hye-Sook Kim, Jeong Hwa Hong, Sung Goo Kang. 2005. Gastrodia elata Blume and an Active Component, p-Hydroxybenzyl Alcohol Reduce Focal Ischemic Brain Injury through Antioxidant Related Gene Expressions. Biological \& Pharmaceutical Bulletin 28:6, 1016-1020. [CrossRef]

11. Xavier Khawaja, Jun Xu, Jin-Jun Liang, James E. Barrett. 2004. Proteomic analysis of protein changes developing in rat hippocampus after chronic antidepressant treatment: Implications for depressive disorders and future therapies. Journal of Neuroscience Research 75:4, 451-460. [CrossRef]

12. S.A Phelan, $X$ Wang, $P$ Wallbrandt, K Forsman-Semb, B Paigen. 2003. Overexpression of Prdx6 reduces $\mathrm{H} 2 \mathrm{O} 2$ but does not prevent diet-induced atherosclerosis in the aortic root. Free Radical Biology and Medicine 35:9, 1110-1120. [CrossRef]

13. Nicole Elizabeth Sparling, Shelley Ann Phelan. 2003. Identification of multiple transcripts for antioxidant protein 2 $(<\mathrm{I}>$ Aop2</I $>$ ): differential regulation by oxidative stress and growth factors. Redox Report 8:2, 87-94. [CrossRef]

14. $\mathrm{K}$ Krapfenbauer. 2003. Aberrant expression of peroxiredoxin subtypes in neurodegenerative disorders. Brain Research 967:1-2, 152-160. [CrossRef]

15. Kurt Krapfenbauer, Byong Chul Yoo, Michael Fountoulakis, Eva Mitrova, Gert Lubec. 2002. Expression patterns of antioxidant proteins in brains of patients with sporadic Creutzfeldt-Jacob disease. ELECTROPHORESIS 23:15, 2541-2547. [CrossRef]

16. Toshimichi Shinohara, Dhirendra P. Singh, Nigar Fatma. 2002. LEDGF, a survival factor, activates stress-related genes. Progress in Retinal and Eye Research 21:3, 341-358. [CrossRef] 